\title{
SELECTIVE ACOUSTIC FOCUSING USING TIME-HARMONIC REVERSAL MIRRORS
}

\author{
CHRISTOPHE HAZARD* AND KARIM RAMDANI ${ }^{\dagger}$
}

\begin{abstract}
A mathematical study of the focusing properties of acoustic fields obtained by a time-reversal process is presented. The case of time-harmonic waves propagating in a nondissipative medium containing sound-soft obstacles is considered. In this context, the so-called D.O.R.T. method (decomposition of the time-reversal operator in French) was recently proposed to achieve selective focusing by computing the eigenelements of the time-reversal operator. The present paper describes a justification of this technique in the framework of the far field model, i.e., for an ideal time-reversal mirror able to reverse the far field of a scattered wave. Both cases of closed and open mirrors, that is, surrounding completely or partially the scatterers, are dealt with. Selective focusing properties are established by an asymptotic analysis for small and distant obstacles.
\end{abstract}

Key words. acoustic scattering, time-reversal, far field operator, small obstacles

AMS subject classifications. 35B40, 35P25, 45A05, 74J20

1. Introduction. Acoustic time-reversal has known in the last few years a significant growth of interest, covering a large number of applications (medical imaging, nondestructive testing, etc.). The main idea of this phenomenon is to take advantage of the reversibility of the wave equation in a nondissipative unknown medium to backpropagate signals to the sources that emitted them. Today, the physical literature (cf. [9] for more details) on this topic is quite rich. Meanwhile, some mathematical works started to deal with different aspects of time-reversal phenomena: see, for instance, $[2,4]$ for time-reversal in the time domain, [14] for time-reversal in the frequency domain, and [15] for time-reversal in random media.

In this work, we present a mathematical analysis of the so-called D.O.R.T. method (decomposition of the time-reversal operator in French), detailed in [16] to achieve selective focusing on diffracting obstacles using time-reversal mirrors (TRM) which are able to emit and receive acoustic waves. In the frequency domain, this method can be described as follows: the TRM first emits an acoustic wave in a homogeneous and nondissipative medium containing some unknown obstacles, and then measures the diffracted field. The measured field is then conjugated (reversing time amounts to a conjugation when the time dependence is of the form $e^{i \omega t}$ ), and re-emitted. The time-reversal operator $T$ is the operator obtained by iterating this procedure twice. The experimental results obtained in [16] show that the number of nonzero (or significant) eigenvalues of $T$ is exactly the number of obstacles contained in the propagation medium. Furthermore, the corresponding eigenvectors generate incident waves that focus selectively on the obstacles. Our aim here is to present a mathematical justification of these results related to selective focusing using TRMs; we will show that these results are not true in general, but do hold for small and distant obstacles with distinct reflectivities.

Let us point out that the inverse problem which consists of recovering the location of the obstacles from the scattering data is not dealt with in this paper. We are mainly concerned with qualitative properties of the eigenvectors of the time-reversal

*Laboratoire SMP, CNRS / ENSTA, 32 Boulevard Victor, 75739 Paris Cedex 15, France (hazard@ ensta.fr).

${ }^{\dagger}$ INRIA (Projet CORIDA) and Institut Elie Cartan, University of Nancy I, POB 239, Vandœuvreles-Nancy, 54506 France (ramdani@loria.fr). 
operator. The eigenvectors corresponding to significant eigenvalues span some kind of relevant subspace in the sense that they contain nearly all the information about the obstacles which can be extracted from the time-reversal operator. The others span the so-called noise subspace, which represents some kind of quasi-null space. This point of view meets the basics of the so-called MUSIC algorithm used in signal processing and imaging, and its generalization, the linear sampling method (LSM), used in inverse scattering (for a short presentation of these methods, see, for instance, [3], and for more details, cf. [12]). These methods answer the inverse problem by using a convenient characterization of the relevant subspace. In the context of scattering, the link between the scattering data and the unknown locations of the obstacles is made by means of point sources: if the radiated field produced by a given point source has a nonzero component in the relevant subspace, the point belongs to one scatterer, otherwise it is outside. But as mentioned in [3], both MUSIC and LSM use the noise subspace: the question of recovering the geometric information directly from the relevant subspace remains open.

The paper is organized as follows. We first deal with a TRM which entirely surrounds the obstacles. In section 2 , we describe the mathematical model used to analyze time-reversal phenomena in the framework of time harmonic waves in the far field model, i.e., for an ideal TRM able to reverse the asymptotic behavior at large distance of a scattered wave. This will in particular lead us to express the timereversal operator by means of the far field operator, well known in scattering theory. Section 3 recalls some results obtained in [14], concerning the global focusing properties of the eigenvectors of the time-reversal operator. The main result of the paper, which concerns selective focusing, is given in section 4. It provides a mathematical justification of the D.O.R.T. method for the problem of scattering by several small and distant obstacles. In section 5, we generalize the results obtained in the previous sections to the case of open mirrors (i.e., mirrors which do not completely surround the scatterers). The main ingredient for the proof of our main result is formula (4.2), which provides the asymptotic behavior of the scattering amplitude for the diffraction by many small obstacles. This formula, which is of independent interest, is proved in the appendix.

2. Mathematical setting of the problem and definition of the timereversal operator. Consider a TRM completely surrounding a collection of soundsoft obstacles, located in a homogeneous medium of celerity $c$. During the emission step, the TRM illuminates the obstacles with an incident wave $u_{I}$ which is supposed to be a Herglotz wave. Such waves are superpositions of planes waves $u_{I}^{\alpha}(x)=\exp (i k \alpha \cdot x)$ of direction $\alpha \in S^{2}\left(S^{2}\right.$ denotes the unit sphere in $\mathbb{R}^{3}, k=\omega / c$ is the wavenumber, and $\omega$ is the frequency). More precisely, given a directional distribution $f \in L^{2}\left(S^{2}\right)$, we suppose that the incident field emitted by the TRM has the form

$$
u_{I}(x)=\int_{S^{2}} f(\alpha) u_{I}^{\alpha}(x) d \alpha=\int_{S^{2}} f(\alpha) e^{i k \alpha \cdot x} d \alpha
$$

We assume that the TRM is located far enough from the obstacles, so that its influence on the diffracted field can be neglected. Moreover, the TRM is supposed to measure the far field corresponding to the diffracted field. 
Let $\Omega$ denote the propagation domain located outside the obstacles and let $\nu$ be the outgoing normal to $\Omega$ on its boundary $\Gamma=\partial \Omega$. When illuminated by the incident plane wave $u_{I}^{\alpha}(x)=e^{i k \alpha \cdot x}$ of direction $\alpha \in S^{2}$, the obstacles generate the diffracted field $u_{D}^{\alpha}$ that solves the classical Dirichlet exterior problem:

$$
\left\{\begin{aligned}
\Delta u_{D}^{\alpha}+k^{2} u_{D}^{\alpha} & =0, \\
u_{D}^{\alpha} & =-u_{I}^{\alpha}, \\
\lim _{R \longrightarrow+\infty} \int_{S_{R}}\left|\frac{\partial u_{D}^{\alpha}}{\partial \nu}-i k u_{D}^{\alpha}\right|^{2} d x & =0,
\end{aligned}\right.
$$

where $S_{R}$ is the sphere $\left\{x \in \mathbb{R}^{3} ;\|x\|=R\right\}$ and where $\partial u_{D}^{\alpha} / \partial \nu$ denotes the radial derivative of $u_{D}^{\alpha}$ on $S_{R}$.

It is well known (cf. [7]) that the far field asymptotics of the diffracted field in a given direction $\beta \in S^{2}$ is given by the formula

$$
u_{D}^{\alpha}(\beta\|x\|)=\frac{e^{i k\|x\|}}{\|x\|} A(\alpha, \beta)+O\left(\|x\|^{-2}\right),
$$

where the bound $O\left(\|x\|^{-2}\right)$ is uniform for all $\beta \in S^{2}$, and where $A(\alpha, \beta)$ is known as the scattering amplitude. This function satisfies some remarkable properties (cf. [7]), which are summarized in the following.

Proposition 2.1. The scattering amplitude $A(\cdot, \cdot)$ is given by the formula

$$
A(\alpha, \beta)=\frac{1}{4 \pi} \int_{\Gamma} \frac{\partial u_{T}^{\alpha}}{\partial \nu}(y) \overline{u_{I}^{\beta}(y)} d \Gamma_{y},
$$

where $u_{T}^{\alpha}=u_{I}^{\alpha}+u_{D}^{\alpha}$ denotes the total field associated with the incident field $u_{I}^{\alpha}$. Furthermore, $A(\cdot, \cdot)$ defines an analytic function on $S^{2} \times S^{2}$ and satisfies the reciprocity relation

$$
A(\alpha, \beta)=A(-\beta,-\alpha)
$$

Remark 1. This reciprocity relation simply states that the behavior of the diffracted field observed in the direction $\beta$ when the scatterers are illuminated by a plane wave of direction $\alpha$, is identical to its behavior in the direction $-\alpha$ under an incident plane wave with direction $-\beta$. This property is a direct consequence of the symmetry of the Green function of the diffraction problem (which follows itself from the self-adjointness of the Dirichlet Laplacian).

Note that in (2.2), the integral actually represents the duality product between $H^{1 / 2}(\Gamma)$ and $H^{-1 / 2}(\Gamma)$ since $\partial u_{T}^{\alpha} / \partial \nu$ belongs to the latter in general. We keep this simplified notation in what follows.

By linearity, it follows from the results above that when illuminated by the Herglotz wave (2.1) associated with a given directional distribution $f \in L^{2}\left(S^{2}\right)$, the scattering obstacles generate the diffracted field $u_{D}$

$$
u_{D}(x)=\int_{S^{2}} f(\alpha) u_{D}^{\alpha}(x) d \alpha .
$$

Furthermore, the asymptotic behavior of $u_{D}$ is given by the formula

$$
u_{D}(\beta\|x\|)=\frac{e^{i k\|x\|}}{\|x\|} F f(\beta)+O\left(\|x\|^{-2}\right),
$$


where the far field $F f(\beta)$ in the direction $\beta \in S^{2}$ is simply given by the relation

$$
F f(\beta)=\int_{S^{2}} A(\alpha, \beta) f(\alpha) d \alpha .
$$

The integral operator $F: L^{2}\left(S^{2}\right) \longrightarrow L^{2}\left(S^{2}\right)$ with kernel $A(\cdot, \cdot)$ is known in the literature as the far field operator. Its properties are given in the following.

Proposition 2.2. The far field operator $F: L^{2}\left(S^{2}\right) \longrightarrow L^{2}\left(S^{2}\right)$ defined by equation (2.4) is a compact and normal operator. Its adjoint is the operator $F^{*}$ : $L^{2}\left(S^{2}\right) \longrightarrow L^{2}\left(S^{2}\right)$ defined by

$$
F^{*} f=\overline{R F \overline{R f}} \quad \forall f \in L^{2}\left(S^{2}\right),
$$

where $R$ is the symmetry operator defined by $R f(\alpha)=f(-\alpha) \forall \alpha \in S^{2}$.

Proof. The compactness of the integral operator $F$ follows immediately from the analyticity of its kernel $A(\cdot, \cdot)$. The fact that $F$ is a normal operator is a well-known result, which is proved, for instance, in [5] (see Corollary 2.5). The adjoint $F^{*}$ of $F$ is the integral operator with kernel

$$
A^{*}(\alpha, \beta)=\overline{A(\beta, \alpha)}=\overline{A(-\alpha,-\beta)},
$$

where we have used the reciprocity relation (2.3). Formula (2.5) follows.

Remark 2. In fact, in [5], it is proved more precisely that

$$
F F^{*}=F^{*} F=\frac{2 \pi}{i k}\left(F-F^{*}\right) .
$$

Since the far field operator $F$ is related to the scattering matrix by the relation $S=I+(i k / 2 \pi) F$, formula (2.6) can be seen as an equivalent formulation of the fact that the scattering operator $S$ is unitary, which is a classical result in scattering theory (cf. [13]).

We are now able to give a rigorous definition of the time-reversal operator. During the time-reversal process, when a Herglotz wave associated with a density $f \in L^{2}\left(S^{2}\right)$ is emitted by the TRM, the far field corresponding to the diffracted field is measured, conjugated, and then re-emitted by the TRM. The new emission is characterized by the Herglotz wave associated with the density $g \in L^{2}\left(S^{2}\right)$ defined by

$$
g=\overline{R F f} .
$$

In this relation, the presence of the symmetry operator $R$ is due to the fact that during the time-reversal process, the far field measured in a given direction $\beta \in S^{2}$ is used to define the new incident plane wave in the direction $-\beta$. The time-reversal operator $T$ is then obtained by iterating this scheme once again, and thus, we have

$$
T f=\overline{R F g}=\overline{R F \overline{R F f}} .
$$

Thanks to (2.5) and using the fact that $F$ is a normal operator, we finally get the following.

Proposition 2.3. The time-reversal operator $T: L^{2}\left(S^{2}\right) \longrightarrow L^{2}\left(S^{2}\right)$ is given by

$$
T=F^{*} F=F F^{*} .
$$

It is the integral operator with kernel

$$
t(\alpha, \beta)=\frac{1}{4 \pi} \int_{\Gamma \times \Gamma} j_{0}(k\|y-z\|) \frac{\partial u_{T}^{\alpha}}{\partial \nu}(y) \overline{\frac{\partial u_{T}^{\beta}}{\partial \nu}(z)} d \Gamma_{y} d \Gamma_{z},
$$


where $u_{T}^{\alpha}=u_{I}^{\alpha}+u_{D}^{\alpha}$ denotes the total field associated with the incident field $u_{I}^{\alpha}$, and $j_{0}(\xi)=\sin (\xi) / \xi$ is the spherical Bessel function of order 0 .

Proof. Since (2.7) has been already proved, we only have to show the second part of the proposition. From (2.7), it follows that $T$ is the integral operator with kernel

$$
t(\alpha, \beta)=\int_{S^{2}} A(\alpha, \gamma) \overline{A(\beta, \gamma)} d \gamma .
$$

Substituting expression (2.2) of the scattering amplitude in the above relations and inverting the integrals over $S^{2}$ with the integrals over $\Gamma$, we find that

$$
t(\alpha, \beta)=\frac{1}{(4 \pi)^{2}} \int_{\Gamma \times \Gamma}\left(\int_{S^{2}} \overline{u_{I}^{\gamma}(y)} u_{I}^{\gamma}(z) d \gamma\right) \frac{\partial u_{T}^{\alpha}}{\partial \nu}(y) \overline{\frac{\partial u_{T}^{\beta}}{\partial \nu}(z)} d \Gamma_{y} d \Gamma_{z} .
$$

Equation (2.8) follows then from the identity (cf. [1, p. 155])

$$
\int_{S^{2}} \overline{u_{I}^{\gamma}(y)} u_{I}^{\gamma}(z) d \gamma=\int_{S^{2}} e^{i k \gamma \cdot(z-y)} d \gamma=4 \pi j_{0}(k\|y-z\|) .
$$

3. Global focusing. The time-reversal operator $T=F^{*} F: L^{2}\left(S^{2}\right) \longrightarrow L^{2}\left(S^{2}\right)$ is clearly a positive and self-adjoint operator. Moreover, by Proposition 2.2, it is also a compact operator. Besides the value 0 , its spectrum is thus constituted of a finite or countable sequence of positive eigenvalues admitting 0 as the only possible accumulation point. In this section, we see how these eigenvectors can be used to generate incident waves that focus acoustic on the diffracting obstacles. These global focusing results (namely Propositions 3.2 and 3.3) actually are a reformulation of results obtained in [14]. First, we recall a classical result from linear operators theory (see, for instance, [20, p. 442]).

Proposition 3.1. Let $N$ be a compact and normal on a Hilbert space $H$. If $\lambda_{1}, \lambda_{2}, \ldots$ is the sequence of all nonzero eigenvalues of $N$, arranged such that $\left|\lambda_{1}\right| \geq$ $\left|\lambda_{2}\right| \geq \cdots$, and if $\varphi_{1}, \varphi_{2}, \ldots$ is a corresponding orthonormal sequence of eigenvectors, then $\left|\lambda_{1}\right|^{2} \geq\left|\lambda_{2}\right|^{2} \geq \cdots$ is the sequence of all nonzero eigenvalues of $N^{*} N=N N^{*}$, and $\varphi_{1}, \varphi_{2}, \ldots$ is a corresponding orthonormal sequence of eigenvectors.

This proposition shows that the nonzero eigenvalues of the time reversal operator $T=F^{*} F=F F^{*}$ are exactly the positive numbers $\left|\lambda_{1}\right|^{2} \geq\left|\lambda_{2}\right|^{2} \geq \cdots$, where the complex numbers $\left(\lambda_{p}\right)_{p \geq 1}$ denote the nonzero eigenvalues of the normal compact far field operator $F$. Furthermore, the corresponding eigenvectors $\left(f_{p}\right)_{p \geq 1}$ of $F$ are exactly the eigenvectors of $T=F^{*} F$. Consequently, it suffices to analyze the focusing properties of the eigenvectors of the far field $F$ to obtain the same results for the time reversal operator $T$.

Let us first deal with the largest eigenvalue of the far field operator. Then, we have the following.

Proposition 3.2. Let $\lambda_{1}$ be the largest eigenvalue (in modulus) of $F$, and let $f_{1} \in L^{2}\left(S^{2}\right)$ be an eigenvector of $F$ associated with $\lambda_{1}$. Then,

$$
\sup _{f \in L^{2}\left(S^{2}\right), f \neq 0} \frac{\|F f\|_{L^{2}\left(S^{2}\right)}^{2}}{\|f\|_{L^{2}\left(S^{2}\right)}^{2}}=\frac{\left\|F f_{1}\right\|_{L^{2}\left(S^{2}\right)}^{2}}{\left\|f_{1}\right\|_{L^{2}\left(S^{2}\right)}^{2}}=\left|\lambda_{1}\right|^{2} .
$$

In other words, the incident Herglotz wave $u_{I}^{1}(x)=\int_{S^{2}} f_{1}(\alpha) e^{i k \alpha \cdot x} d \alpha$ is, among all the possible Herglotz waves, the one that maximizes the energy scattered by the obstacles. 
Proof. The proposition is a straightforward consequence of the Min-Max principle. Indeed, applying this principle to the positive self-adjoint and bounded operator $T=$ $F^{*} F$, we can write that the largest eigenvalue $\left|\lambda_{1}\right|^{2}$ of $T$ satisfies

$$
\left|\lambda_{1}\right|^{2}=\sup _{f \in L^{2}\left(S^{2}\right), f \neq 0} \frac{(T f, f)_{L^{2}\left(S^{2}\right)}}{\|f\|_{L^{2}\left(S^{2}\right)}^{2}}=\sup _{f \in L^{2}\left(S^{2}\right), f \neq 0} \frac{\|F f\|_{L^{2}\left(S^{2}\right)}^{2}}{\|f\|_{L^{2}\left(S^{2}\right)}^{2}} .
$$

Roughly speaking, this result says that the "best" way to illuminate a family of obstacles with Herglotz waves is to use a Herglotz wave $u_{I}^{1}$ corresponding to an eigenvector $f_{1}$ of $F$ (or $T$ ) associated with its largest eigenvalue $\lambda_{1}$. The physical reason explaining this property is that the incident field generated by an eigenvector $f_{p}$ associated with any eigenvalue $\lambda_{p} \neq 0$ of $F$, focuses on the obstacles. More precisely, the following result holds true (see [14]).

Proposition 3.3. Let $\lambda_{p} \neq 0$ be an eigenvalue of $F$ and $f_{p} \in L^{2}\left(S^{2}\right), f_{p} \neq 0$, an eigenvector of $F$ associated with $\lambda_{p}$. Then, the Herglotz wave $u_{I, p}$ associated with $f_{p}$ and defined by $u_{I, p}(x)=\int_{S^{2}} f_{p}(\alpha) u_{I}^{\alpha}(x) d \alpha=\int_{S^{2}} f_{p}(\alpha) e^{i k \alpha \cdot x} d \alpha$, has the following form:

$$
u_{I, p}(x)=\frac{1}{\lambda_{p}} \int_{\Gamma} j_{0}(k\|x-y\|) \frac{\partial u_{T, p}}{\partial \nu}(y) d \Gamma_{y},
$$

where $u_{T, p}=u_{I, p}+u_{D, p}$ denotes the total field associated with the incident field $u_{I, p}$.

Proof. Since $f_{p}(\beta)=\lambda_{p}^{-1} F f_{p}(\beta)=\lambda_{p}^{-1} \int_{S^{2}} A(\alpha, \beta) f_{p}(\alpha) d \alpha$, we obtain by using expression (2.2) of $A(\alpha, \beta)$ that

$$
\begin{aligned}
f_{p}(\beta) & =\left(4 \pi \lambda_{p}\right)^{-1} \int_{S^{2}} \int_{\Gamma} \frac{\partial u_{T}^{\alpha}}{\partial \nu} \overline{u_{I}^{\beta}} d \Gamma f_{p}(\alpha) d \alpha \\
& =\left(4 \pi \lambda_{p}\right)^{-1} \int_{\Gamma} \int_{S^{2}} \frac{\partial u_{T}^{\alpha}}{\partial \nu} f_{p}(\alpha) d \alpha \overline{u_{I}^{\beta}} d \Gamma .
\end{aligned}
$$

But by superposition, the integral $\int_{S^{2}} \partial u_{T}^{\alpha} / \partial \nu f_{p}(\alpha) d \alpha$ is nothing but the normal derivative of the total field $u_{T, p}$ associated with the incident field $u_{I, p}$, and thus

$$
f_{p}(\beta)=\left(4 \pi \lambda_{p}\right)^{-1} \int_{\Gamma} \frac{\partial u_{T, p}}{\partial \nu} \overline{u_{I}^{\beta}} d \Gamma .
$$

We can now obtain the expression of the incident field generated by the eigenvector $f_{p}$. From (3.2), we have

$$
\begin{aligned}
u_{I, p}(x) & =\int_{S^{2}} f_{p}(\beta) u_{I}^{\beta}(x) d \beta \\
& =\left(4 \pi \lambda_{p}\right)^{-1} \int_{S^{2}} \int_{\Gamma} \frac{\partial u_{T, p}}{\partial \nu} \overline{u_{I}^{\beta}} d \Gamma u_{I}^{\beta}(x) d \beta \\
& =\left(4 \pi \lambda_{p}\right)^{-1} \int_{\Gamma}\left(\int_{S^{2}} u_{I}^{\beta}(x) \overline{u_{I}^{\beta}(y)} d \beta\right) \frac{\partial u_{T, p}}{\partial \nu}(y) d \Gamma_{y} .
\end{aligned}
$$

Formula (3.1) follows then from identity (2.10).

Since $j_{0}(\xi)=\sin (\xi) / \xi$, formula (3.1) shows that, as expected, the incident field $u_{I, p}(x)$ generated by an eigenvector $f_{p}$ of $F$ (or $T$ ) decreases like $r(x)^{-1}$ if $r(x)$ denotes the distance of $x$ to the obstacles. In this sense, one can say that $u_{I, p}$ focuses on the obstacles located in the propagation medium. Furthermore, the quality of this 
focusing (given by the amplitude of the far field) is exactly given by the magnitude of the eigenvalue $\lambda_{p}$, since

$$
\left|\lambda_{p}\right|=\frac{\left\|F f_{p}\right\|_{L^{2}\left(S^{2}\right)}}{\left\|f_{p}\right\|_{L^{2}\left(S^{2}\right)}} .
$$

4. Selective focusing. The aim of this section is to propose a mathematical justification of the so-called D.O.R.T. method presented in [16] and briefly described in the introduction of this paper. Roughly speaking, we answer the two following questions.

(i) Is the number of obstacles contained in a homogeneous medium equal to the number of "significant" eigenvalues of the far field operator $F$ (or, equivalently, to those of the time-reversal operator $\left.T=F^{*} F=F F^{*}\right)$ ?

(ii) If so, do the associated eigenvectors selectively focus on the obstacles?

As can be seen from the numerical experiments presented in [6], the answer to the first question is, in general, negative (there can be several "significant" eigenvalues even when there is just one scatterer). We will confirm this result by studying in subsection 4.1 the special case of a single spherical obstacle. Nevertheless, we will show that the answer becomes positive provided the obstacles considered are small enough. Under this assumption, we show in subsection 4.2 that selective focusing can be achieved using the eigenvectors of the far field operator.

4.1. Diffraction by a single spherical obstacle. In this subsection, we deal with the case where the scatterer is a sphere of radius $a>0$. For this particular geometry, an explicit formula can be obtained for the eigenvalues of the far field mapping and thus for those of the time-reversal operator. The results of this subsection are classical and can be found, for instance, in [7]. In particular, formula (3.30) in [7] shows that for any given density

$$
f=\sum_{n=0}^{+\infty} \sum_{m=-n}^{n} a_{n}^{m} Y_{n}^{m} \in L^{2}\left(S^{2}\right)
$$

we have

$$
F f(\beta)=\sum_{n=0}^{+\infty} \sum_{m=-n}^{n} \frac{4 i \pi}{k} \frac{j_{n}(k a)}{h_{n}^{1}(k a)} a_{n}^{m} Y_{n}^{m}(\beta)
$$

Here $Y_{n}^{m}$ denotes the usual spherical harmonics, $j_{n}$ and $h_{n}^{1}$ are, respectively, the spherical Bessel and Hankel functions of order $n$.

Since the spherical harmonics constitute an orthonormal basis of $L^{2}\left(S^{2}\right)$, this formula shows that the following result holds.

Proposition 4.1. The eigenvalues of the far field operator $F$ in the case of a single sound-soft spherical scatterer of radius a are given by

$$
\lambda_{n}=\frac{4 i \pi}{k} \frac{j_{n}(k a)}{h_{n}^{1}(k a)} \quad \forall n \geq 1 .
$$

The eigenspace associated with the eigenvalue $\lambda_{n}$ is the vector space of dimension $2 n+1$ with basis $Y_{n}^{m}$, for $|m| \leq n$.

Remark 3. Equation (4.1) shows that the eigenvalues $\lambda_{n}$ of the far field operator $F$ satisfy $\left|\lambda_{n}\right| \leq 4 \pi / k$ (recall that $h_{n}^{1}=j_{n}+i y_{n}$ ). This property can also be obtained 


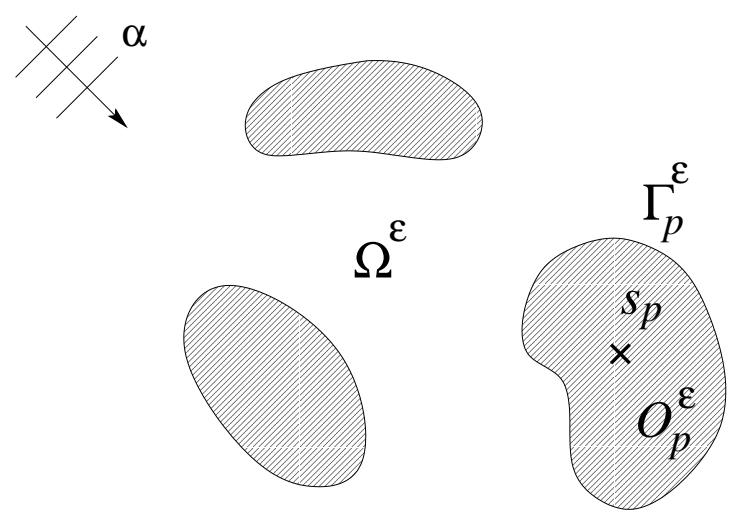

FIG. 4.1.

from the fact that the scattering operator $S=I+(i k / 2 \pi) F$ is unitary. Indeed, this property implies that the eigenvalues $\lambda_{n}$ lie on the circle of radius $4 \pi / k$ centered at $(0,2 \pi / k)$.

Proposition 4.1 shows in particular that the number of nonzero eigenvalues is not necessarily equal to the number of obstacles. However, in the case of a point scatterer or in the case of the low-frequency scattering (both cases which correspond to the asymptotic limit $k a \longrightarrow 0$ ), this result becomes true. Indeed, using the asymptotic behavior of Bessel and Hankel functions, we easily see that the eigenvalues $\lambda_{n}$ given by (4.1) satisfy

$$
\lambda_{n} \sim-\frac{4 \pi^{2}}{k} \frac{(k a / 2)^{2 n+1}}{\Gamma(n+1 / 2) \Gamma(n+3 / 2)}
$$

when $k a$ goes to zero (and $n$ is fixed). Thus, $\lambda_{n+1} / \lambda_{n}$ decreases like $(k a)^{2}$, and hence, one can consider that the only significant eigenvalue in the limit case $k a \longrightarrow 0$ is the largest one $\lambda_{1}$. This observation suggests that the number of nonzero eigenvalues can be related to the number of obstacles when the obstacles are small. The next subsection provides a justification of this statement.

4.2. Diffraction by several small obstacles. Consider a family of obstacles $\left\{\mathcal{O}_{p}^{\varepsilon} ; p=1, N\right\}$ depending on a small parameter $\varepsilon$, where each $\mathcal{O}_{p}^{\varepsilon}$ is the image of a reference open set $\mathcal{O}_{p}$ (which is assumed to contain the origin) by a dilation of ratio $\varepsilon$ centered at a given point $s_{p} \in \mathbb{R}^{3}$ (see Figure 4.1):

$$
\mathcal{O}_{p}^{\varepsilon}=\left\{x \in \mathbb{R}^{3} ; \xi=\frac{x-s_{p}}{\varepsilon} \in \mathcal{O}_{p}\right\}
$$

Of course the "centers" $s_{p}$ are chosen different so that for small enough $\varepsilon$, the obstacles do not intersect.

The main ingredient to show that selective focusing can be achieved using the eigenvectors of the far field operator when $\varepsilon$ is small enough is given by the following result, which provides the asymptotic behavior of the scattering amplitude $A^{\varepsilon}(\alpha, \beta)$ associated with the family of obstacles $\left\{\mathcal{O}_{p}^{\varepsilon}\right\}$.

Proposition 4.2. There exist $N$ positive constants $C_{1}, \ldots, C_{N}$ depending only on the geometry of the reference obstacles $\mathcal{O}_{1}, \ldots, \mathcal{O}_{N}$ (called the "capacities" of these 
obstacles) such that

$$
\frac{A^{\varepsilon}(\alpha, \beta)}{\varepsilon}=A^{(1)}(\alpha, \beta)+O(\varepsilon) \text { with } A^{(1)}(\alpha, \beta)=\frac{-1}{4 \pi} \sum_{p=1, N} C_{p} u_{I}^{\alpha}\left(s_{p}\right) \overline{u_{I}^{\beta}\left(s_{p}\right)}
$$

where the bound $O(\varepsilon)$ is uniform for all $\alpha, \beta \in S^{2}$.

For the sake of clarity, the - rather technical-proof of this proposition is given in the appendix.

Remark 4 . The capacity of a spherical soft obstacle of radius $a$ is $C=4 \pi a$ (since the solution to (A.7) is simply given in this case by $V(x)=a /\|x\|)$.

Thanks to Proposition 4.2, we know that the far field operator $F^{\varepsilon}$ of the family of obstacles $\left\{\mathcal{O}_{p}^{\varepsilon} ; p=1, N\right\}$ satisfies

$$
\left\|\varepsilon^{-1} F^{\varepsilon}-F^{(1)}\right\|_{\mathcal{L}\left(L^{2}\left(S^{2}\right)\right)}=\sup _{f \in L^{2}\left(S^{2}\right) \backslash\{0\}} \frac{\left\|\left(\varepsilon^{-1} F^{\varepsilon}-F^{(1)}\right) f\right\|_{L^{2}\left(S^{2}\right)}}{\|f\|_{L^{2}\left(S^{2}\right)}}=O(\varepsilon),
$$

where $F^{(1)}$ is the integral operator on $L^{2}\left(S^{2}\right)$ with kernel $A^{(1)}$ :

$$
F^{(1)} f(\beta)=\int_{S^{2}} A^{(1)}(\alpha, \beta) f(\alpha) d \alpha
$$

Since $F^{\varepsilon}$ is compact and normal, perturbation theory [11] ascertains the continuity of any finite system of eigenvalues as well as of the associated total eigenprojection. More precisely, assume that $\lambda^{(1)}$ is an isolated eigenvalue of $F^{(1)}$ with finite multiplicity $m$, which implies that $\lambda^{(1)} \neq 0$.

(i) Then for small enough $\varepsilon$, the spectrum of $\varepsilon^{-1} F^{\varepsilon}$ can be separated into two parts. On one hand, the so-called $\lambda^{(1)}$-group consists of $m^{\prime} \leq m$ eigenvalues $\lambda_{j}^{\varepsilon}$, with $j=1$ to $m^{\prime}$, having a constant multiplicity $m_{j}$ for $\varepsilon \neq 0$, and which are continuous near $\varepsilon=0$, namely

$$
\left|\lambda_{j}^{\varepsilon}-\lambda^{(1)}\right|=O(\varepsilon)
$$

Moreover, the total multiplicity $\sum_{j=1, m^{\prime}} m_{j}$ of the $\lambda^{(1)}$-group coincide with the multiplicity $m$ of $\lambda^{(1)}$. On the other hand, the complementary of the $\lambda^{(1)}$-group in the spectrum of $\varepsilon^{-1} F^{\varepsilon}$ lies outside a vicinity of $\lambda^{(1)}$.

(ii) The total projection $P^{\varepsilon}$ for the $\lambda^{(1)}$-group, i.e., the sum of the orthogonal projections on the eigenspaces associated with the $\lambda_{j}^{\varepsilon}$, is continuous at $\varepsilon=0$, and

$$
\left\|P^{\varepsilon}-P^{(1)}\right\|_{\mathcal{L}\left(L^{2}\left(S^{2}\right)\right)}=O(\varepsilon)
$$

where $P^{(1)}$ is the eigenprojection associated with $\lambda^{(1)}$.

Notice that in general, one cannot assert the existence of a continuous family of eigenvectors associated respectively with the $\lambda_{j}^{\varepsilon}$. However, for our particular choice of geometric perturbation ( $\varepsilon$-dilation), such a result holds, since the perturbation actually is analytic with respect to $\varepsilon$ (which is easily deduced from the appendix). But this result is of poor practical interest.

An eigenvalue of $\varepsilon^{-1} F^{\varepsilon}$ either belongs to some $\lambda^{(1)}$-group for a nonzero eigenvalue $\lambda^{(1)}$ of $F^{(1)}$, or vanishes as $\varepsilon$ tends to 0 . In the latter case, the above result does not apply; perturbation theory only provides the continuity of nonstationary eigenelements. So it remains to study the spectral properties of $F^{(1)}$, whose degenerate kernel 
will be rewritten in the form

$$
A^{(1)}(\alpha, \beta)=-\sum_{p=1, N} C_{p} \overline{e_{p}(\alpha)} e_{p}(\beta), \text { where } e_{p}(\alpha)=\frac{e^{-i k \alpha \cdot s_{p}}}{2 \sqrt{\pi}}(p=1, N) .
$$

Remark 5. Each $e_{p}$ appears as a normalized function of $L^{2}\left(S^{2}\right)$ corresponding to an incident Herglotz wave $u_{I, p}$ which focuses on the $p$ th obstacle, for

$$
u_{I, p}(x)=\int_{S^{2}} e_{p}(\alpha) u_{I}^{\alpha}(x) d \alpha=2 \sqrt{\pi} j_{0}\left(k\left\|x-s_{p}\right\|\right),
$$

by virtue of (2.10).

The above expression of $A^{(1)}$ then yields

$$
F^{(1)} f=-\sum_{p=1, N} C_{p}\left(f, e_{p}\right)_{L^{2}\left(S^{2}\right)} e_{p}
$$

Proposition 4.3. The limit far field operator (4.4) is a negative self-adjoint operator with finite rank $N$ (the number of obstacles) and whose spectral radius cannot be smaller than the greatest capacity $C_{p}$ of the obstacles.

In the case where the wavelength $\ell=2 \pi / k$ is small compared with the minimum distance $d=\min _{1 \leq p \neq q \leq N}\left\|s_{p}-s_{q}\right\|$ between the obstacles, the family $\left\{e_{p} ; p=1, N\right\}$ defined in (4.3) provides an approximate basis of eigenvectors associated with the approximate eigenvalues $\left\{-C_{p} ; p=1, N\right\}$ :

$$
F^{(1)} e_{p}=-C_{p} e_{p}+O\left(\frac{\ell}{d}\right) .
$$

Proof. The bilinear form associated with $F^{(1)}$,

$$
\left(F^{(1)} f, f^{\prime}\right)_{L^{2}\left(S^{2}\right)}=-\sum_{p=1, N} C_{p}\left(f, e_{p}\right)_{L^{2}\left(S^{2}\right)} \overline{\left(f^{\prime}, e_{p}\right)_{L^{2}\left(S^{2}\right)}},
$$

is clearly negative and self-adjoint, and so is $F^{(1)}$. The range of $F^{(1)}$ is spanned by $\left\{e_{p} ; p=1, N\right\}$. To see that this family is linearly independent, suppose that

$$
\sum_{p=1, N} z_{p} e_{p}=0 \text { with } z_{p} \in \mathbb{C} .
$$

It is clear that the function $e_{p} \in L^{2}\left(S^{2}\right)$ is nothing but the far field corresponding to a point source located at the point $s_{p}$. Consequently, the above relation simply states that we have chosen a superposition of point sources located at the points $\left(s_{p}\right)_{p=1, N}$ whose far field vanishes. Thus, by Rellich's lemma, the field is identically zero. Hence, all the coefficients $\left(z_{p}\right)_{p=1, N}$ of the linear combination must also vanish. The linear independence of the family $\left\{e_{p} ; p=1, N\right\}$ is thus established.

The lower bound for the spectral radius follows from the fact that

$$
\left|\left(F^{(1)} e_{q}, e_{q}\right)_{L^{2}\left(S^{2}\right)}\right|=\sum_{p=1, N} C_{p}\left|\left(e_{p}, e_{q}\right)_{L^{2}\left(S^{2}\right)}\right|^{2} \geq C_{q} \text { for } q=1, N,
$$

since the $e_{p}$ are normalized in $L^{2}\left(S^{2}\right)$. On the other hand, nothing can be said in general about the gap between 0 and the other eigenvalues, which may be arbitrarily 
close to the former. This actually depends on the constructive or destructive interactions between the different obstacles, which are measured by the following scalar products (see (2.10)):

$$
\left(e_{p}, e_{q}\right)_{L^{2}\left(S^{2}\right)}=j_{0}\left(k\left\|s_{p}-s_{q}\right\|\right)=\frac{\sin \left(k\left\|s_{p}-s_{q}\right\|\right)}{k\left\|s_{p}-s_{q}\right\|} .
$$

These relations show in particular that

$$
\left(e_{p}, e_{q}\right)_{L^{2}\left(S^{2}\right)}=\left\{\begin{array}{cc}
1 & \text { for } q=p, \\
O\left(\frac{\ell}{d}\right) & \text { for } q \neq p,
\end{array}\right.
$$

which means that $\left\{e_{p} ; p=1, N\right\}$ is close to an orthogonal basis of the range of $F^{(1)}$ when $\ell \ll d$. The estimate (4.5) follows: each $e_{p}$ is an approximate eigenvector.

What are the practical consequences of the above results as regards selective focusing? Mainly that the eigenvectors of the time-reversal operator (or the far field operator) will produce selective focusing acoustic waves if

(i) the obstacles are small enough, compared to the wavelength,

(ii) the smallest distance between them is large, compared again to the wavelength,

(iii) their capacities are all distinct.

Indeed in this case all the nonzero eigenvalues of $F^{(1)}$ will be simple: the diagonalization of the time-reversal operator will then yield approximations of the focusing densities $e_{p}$.

But if one of these assumptions is missing, the nice focusing properties will disappear, at least for some groups of eigenvectors.

On one hand, if the interactions between the obstacles become significant, i.e., when $d / \ell=O(1)$, these properties may reduce to the purely global focusing presented in section 3. In particular, for very low frequencies, the situation $\varepsilon \ll d \ll \ell$ may occur. In this case we have

$$
e_{p}=\tilde{e}+O\left(\frac{d}{\ell}\right) \quad \text { with } \tilde{e}(\alpha)=\frac{e^{-i k \alpha \cdot \tilde{s}}}{2 \sqrt{\pi}},
$$

where $\tilde{s}$ may be chosen as a convex combination of the $s_{p}$. As a consequence

$$
F^{(1)} f=-\left(\sum_{p=1, N} C_{p}\right)(f, \tilde{e})_{L^{2}\left(S^{2}\right)} \tilde{e}+O\left(\frac{d}{\ell}\right),
$$

which shows that the cluster of obstacles behaves like a unique obstacle which accumulates their respective capacities; only one significant eigenvalue of the time-reversal operator may be observed. Of course, for several distant clusters, we shall recover selective focusing on each cluster.

On the other hand, if some of the obstacles have neighboring capacities, the time-reversal operator may admit nonsimple eigenvalues. In this situation, the diagonalization of the latter cannot choose the selective focusing densities among all their linear combinations which compose the corresponding eigenspace.

5. Open time-reversal mirrors. In this section, we consider the case of a TRM that does not entirely surround the obstacle. Given a subset $\widehat{S}$ of $S^{2}$, we assume that 
the TRM can emit plane waves of directions $\alpha \in \widehat{S}$, and measures the far field in the opposite directions $\beta \in(-\widehat{S})$. One emission-diffraction-reception cycle is described by the directional far field operator

$$
\widehat{F}=\widehat{P}_{-} F \widehat{P}_{+}^{*}: L^{2}(+\widehat{S}) \longrightarrow L^{2}(-\widehat{S}),
$$

where $\widehat{P}_{ \pm}$are the restriction operators from $L^{2}\left(S^{2}\right)$ to $L^{2}( \pm \widehat{S})$, and thus their respective adjoints $\widehat{P}_{ \pm}^{*}: L^{2}( \pm \widehat{S}) \longrightarrow L^{2}\left(S^{2}\right)$ are the operators of continuation by 0 outside $\pm \widehat{S}$. Note here that $\widehat{F}$ appears as the integral operator

$$
\widehat{F} f(\beta)=\int_{+\widehat{S}} A(\alpha, \beta) f(\alpha) d \alpha \text { for } \beta \in-\widehat{S}
$$

The time-reversal operator $\widehat{T}$ in the case of an open TRM is then defined by

$$
\widehat{T} f=\overline{\widehat{R} \widehat{F} \overline{\widehat{R} \widehat{F} f}}
$$

where $\widehat{R}: L^{2}(-\widehat{S}) \longrightarrow L^{2}(+\widehat{S})$ is the restriction of the symmetry operator defined in section 2 (i.e., $\widehat{R} f(\alpha)=f(-\alpha)$ for $\alpha \in \widehat{S})$.

But one can easily check that $\widehat{R} \widehat{P}_{-}=\widehat{P}_{+} R$ and $\widehat{P}_{+}^{*} \widehat{R}=R \widehat{P}_{-}^{*}$, and since these operators commute with the conjugation, we have by virtue of $(2.5)$

$$
\widehat{F}^{*} f=\widehat{P}_{+} F^{*} \widehat{P}_{-}^{*} f=\overline{\widehat{P}_{+} R F \overline{R \widehat{P}_{-}^{*} f}}=\overline{\widehat{R} \widehat{P}_{-} F \widehat{P}_{+}^{*} \bar{R} f}=\overline{\widehat{R} \widehat{F} \overline{\widehat{R} f}}
$$

Hence, we can state the following result.

Proposition 5.1. The time-reversal operator $\widehat{T}$ for an open TRM is given by

$$
\widehat{T}=\widehat{F}^{*} \widehat{F}: L^{2}(\widehat{S}) \longrightarrow L^{2}(\widehat{S}) .
$$

Thus, it is the integral operator with kernel

$$
\widehat{t}(\alpha, \beta)=\int_{-\widehat{S}} A(\alpha, \gamma) \overline{A(\beta, \gamma)} d \gamma \text { for } \alpha, \beta \in \widehat{S} .
$$

Moreover, $\widehat{T}$ defines a compact positive and self-adjoint operator.

Besides the value 0 , the spectrum $\widehat{T}$ is thus constituted of a finite or countable sequence of positive eigenvalues $\left(\widehat{\mu}_{p}\right)_{p \geq 1}$ admitting 0 for only possible accumulation point. The largest eigenvalue $\widehat{\mu}_{1}$ of $\widehat{T}$ is thus given by

$$
\widehat{\mu}_{1}=\sup _{f \in L^{2}(\widehat{S}), f \neq 0} \frac{(\widehat{T} f, f)_{L^{2}(\widehat{S})}}{\|f\|_{L^{2}\left(S^{2}\right)}^{2}}=\sup _{f \in L^{2}(\widehat{S}), f \neq 0} \frac{\|\widehat{F} f\|_{L^{2}(-\widehat{S})}^{2}}{\|f\|_{L^{2}(+\widehat{S})}^{2}} .
$$

This expression shows in particular that the incident field corresponding to an eigenvector associated with this eigenvalue maximizes the diffracted field in the direction of the TRM. Our goal now is to see if the global and selective properties proved respectively in sections 3 and 4 for closed mirrors still hold in the case of an open TRM. The 
main difference between both situations is that in the latter one, the directional far field operator $\widehat{F}$ is not anymore normal (the range of $\widehat{F}^{*} \widehat{F}$ is contained in $L^{2}(\widehat{S})$, when that of $\widehat{F} \widehat{F}^{*}$ is contained in $\left.L^{2}(-\widehat{S})\right)$. Consequently, the eigenelements of $\widehat{T}=\widehat{F}^{*} \widehat{F}$ cannot be directly related to those of $\widehat{F}$. Contrary to the case of a closed TRM, the spectral analysis need thus to be carried on the time reversal operator and not on the far field one. Nevertheless, as we are going to see now, all the focusing results obtained previously still hold.

5.1. Global focusing. In this subsection, we prove a global focusing property similar to the one given in Proposition 3.3. More precisely, we have the following result.

Proposition 5.2. Let $\widehat{\mu}_{p} \neq 0$ be an eigenvalue of $\widehat{T}$ and $\widehat{f}_{p} \in L^{2}(\widehat{S})$ be a corresponding eigenvector. Then, the Herglotz wave $\widehat{u}_{I, p}$ associated with $\widehat{f}_{p}$ and defined by $\widehat{u}_{I, p}(x)=\int_{\widehat{S}} \widehat{f}_{p}(\alpha) u_{I}^{\alpha}(x) d \alpha$ can be written in the form

$$
\widehat{u}_{I, p}(x)=\int_{\Gamma} \widehat{\jmath}(k(x-y)) h_{p}(y) d \Gamma
$$

for some density $h_{p}$, where

$$
\widehat{\jmath}(k(x-y))=\int_{\widehat{S}} u_{I}^{\beta}(x) \overline{u_{I}^{\beta}(y)} d \beta=\int_{\widehat{S}} e^{i k \beta \cdot(x-y)} d \beta .
$$

Proof. Like in the proof of Proposition 3.3, formula (5.2) will be proved if we can write $\widehat{f}_{p}$ in the form

$$
\widehat{f}_{p}(\beta)=\int_{\Gamma} h_{p} \overline{u_{I}^{\beta}} d \Gamma
$$

for a given density $h_{p}$. Indeed, if such a relation holds, then

$$
\widehat{u}_{I, p}(x)=\int_{\widehat{S}} \widehat{f}_{p}(\beta) u_{I}^{\beta}(x) d \beta=\int_{\widehat{S}} \int_{\Gamma} h_{p} \overline{u_{I}^{\beta}} d \Gamma u_{I}^{\beta}(x) d \beta .
$$

Equation (5.2) follows then by inverting the integrals over $\widehat{S}$ and $\Gamma$.

Thus, it only remains to prove (5.4). We first write that for all $\beta \in \widehat{S}$,

$$
\widehat{f}_{p}(\beta)=\frac{1}{\widehat{\mu}_{p}} \widehat{T} \widehat{f}_{p}(\beta)=\frac{1}{\widehat{\mu}_{p}} \int_{\widehat{S}} \widehat{t}(\alpha, \beta) \widehat{f}_{p}(\alpha) d \alpha .
$$

Thanks to the reciprocity relation (2.3), formula (5.1) can be written

$$
\widehat{t}(\alpha, \beta)=\int_{-\widehat{S}} A(\alpha, \gamma) \overline{A(-\gamma,-\beta)} d \gamma
$$

Using the integral representation (2.2) in the above relation, we get after some simple computations that

$$
\widehat{t}(\alpha, \beta)=\int_{\Gamma} h_{p}^{\alpha} \overline{u_{I}^{\beta}} d \Gamma
$$


where the density $h_{p}^{\alpha}$ is given by

$$
h_{p}^{\alpha}(x)=\frac{1}{(4 \pi)^{2}} \int_{-\widehat{S}} \int_{\Gamma} \frac{\partial u_{T}^{\alpha}}{\partial \nu} \overline{u_{I}^{\gamma}} d \Gamma \overline{\frac{\partial u_{T}^{-\gamma}}{\partial \nu}(x)} d \gamma .
$$

Combining (5.5) and (5.6), one obtains the claimed relation (5.4), with the density $h_{p}(x)=\widehat{\mu}_{p}^{-1} \int_{\widehat{S}} h_{p}^{\alpha}(x) \widehat{f}_{p}(\alpha) d \alpha$.

It is well known in oscillatory integrals theory that the function $\widehat{\jmath}(x)$ defined by (5.3) satisfies (one can use the stationary phase theorem; see, for instance, Theorem 1 in $[19$, p. 322])

$$
\widehat{\jmath}(x)=\mathcal{O}\left(\|x\|^{-1}\right) .
$$

In the directions which are not covered by the TRM (i.e., when $x /\|x\| \notin \pm S$ ), one can in fact obtain a faster decay for $\widehat{\jmath}(x)$, since we have then $\widehat{\jmath}(x)=\mathcal{O}\left(\|x\|^{-3 / 2}\right)$.

Thanks to (5.7), formula (5.2) shows thus that the incident field generated by an eigenvector of $\widehat{T}$ focuses on the obstacles located in the propagation medium.

5.2. Diffraction by several small obstacles. Now we turn to the analysis of the selective focusing in the case of a TRM partially surrounding several small obstacles. The assumptions made on the geometry of the small scatterers are identical to those made in section 4 . Let us recall that the main difference with section 4 is that since $\widehat{F}$ is not normal, the spectral analysis can no longer be achieved on $\widehat{F}$ but has to be carried out directly on the time-reversal operator $\widehat{T}$. In this subsection, we are going to see that the selective focusing results obtained in section 4 can be extended to the case of an open mirror.

Using the asymptotic formula (4.2) of the scattering amplitude, one easily gets that the kernel $\widehat{t}^{\varepsilon}(\alpha, \beta)$ of the time-reversal operator $\widehat{T}^{\varepsilon}=\left(\widehat{F}^{\varepsilon}\right)^{*} \widehat{F}^{\varepsilon}$ satisfies

$$
\frac{\widehat{t}^{\varepsilon}(\alpha, \beta)}{\varepsilon^{2}}=\widehat{t}^{(1)}(\alpha, \beta)+O(\varepsilon),
$$

where

$$
\widehat{t}^{(1)}(\alpha, \beta)=\int_{-\widehat{S}} A^{(1)}(\alpha, \gamma) \overline{A^{(1)}(\beta, \gamma)} d \gamma \quad \forall \alpha, \beta \in \widehat{S}
$$

and where $A^{(1)}(\cdot, \cdot)$ is the degenerate kernel defined in (4.2). Since $\widehat{T}^{\varepsilon}$ is compact and self-adjoint, classical results of perturbation theory show again that for small $\varepsilon$, the spectral elements of $\varepsilon^{-2} \widehat{T}^{\varepsilon}$ can be approximated by those of the integral operator $\widehat{T}^{(1)}$ with kernel $\widehat{t}^{(1)}(\cdot, \cdot)$, which also reads $\widehat{T}^{(1)}=\left(\widehat{F}^{(1)}\right)^{*} \widehat{F}^{(1)}$, where the operator $\widehat{F}^{(1)}: L^{2}(\widehat{S}) \longrightarrow L^{2}(-\widehat{S})$ is defined by

$$
\widehat{F}^{(1)} f(\beta)=-\sum_{p=1, N} C_{p}\left(f, e_{p}\right)_{L^{2}(\widehat{S})} e_{p}(\beta) \quad \text { for } \beta \in-\widehat{S} .
$$

If we define the normalized functions $\left\{\widehat{e}_{p} ; p=1, N\right\}$ in $L^{2}(\widehat{S})$ and $L^{2}(-\widehat{S})$ by

$$
\widehat{e}_{p}(\alpha)=(4 \pi \widehat{r})^{-1 / 2} e^{-i k \alpha \cdot s_{p}},
$$

where $\widehat{r}=\operatorname{mes}(\widehat{S}) /(4 \pi)$ is the opening ratio of the TRM, then

$$
\widehat{F}^{(1)} f=-\widehat{r} \sum_{p=1, N} C_{p}\left(f, \widehat{e}_{p}\right)_{L^{2}(\widehat{S})} \widehat{e}_{p} \text { in } L^{2}(-\widehat{S}) .
$$


Hence, for all $f \in L^{2}(\widehat{S})$, we have

$$
\widehat{T}^{(1)} f=\widehat{r}^{2} \sum_{q=1, N} C_{q}\left(\sum_{p=1, N} C_{p}\left(f, \widehat{e}_{p}\right)_{L^{2}(\widehat{S})}\left(\widehat{e}_{p}, \widehat{e}_{q}\right)_{L^{2}(-\widehat{S})}\right) \widehat{e}_{q} .
$$

We can now state the main result of this subsection.

Proposition 5.3. The limit time reversal operator $\widehat{T}^{(1)}: L^{2}(\widehat{S}) \longrightarrow L^{2}(\widehat{S})$ defined by (5.10) is a self-adjoint operator with finite rank $N$ (the number of obstacles).

Furthermore, if the wavelength $\ell=2 \pi / k$ is small compared with the minimum distance between the obstacles, the family $\left\{\widehat{e}_{p} ; p=1, N\right\}$ defined in (5.8) provides an approximate basis of eigenvectors of $\widehat{T}^{(1)}$ associated with the approximate eigenvalues $\left(\widehat{r} C_{p}\right)^{2}$ :

$$
\widehat{T}^{(1)} \widehat{e}_{p}=\left(\widehat{r} C_{p}\right)^{2} \widehat{e}_{p}+O\left(\frac{\ell}{d}\right)
$$

Proof. The fact that $\widehat{T}^{(1)}$ is of rank $N$ follows from the fact that the family $\left\{\widehat{e}_{p} ; p=1, N\right\}$ is linearly independent in $L^{2}(\widehat{S})$ (see the proof of Proposition 5.3). Equation (5.11) follows from (5.10) combined with the fact that

$$
\left(\widehat{e}_{p}, \widehat{e}_{q}\right)_{L^{2}(\widehat{S})}=\overline{\left(\widehat{e}_{p}, \widehat{e}_{q}\right)_{L^{2}(-\widehat{S})}}=\left\{\begin{array}{cl}
1 & \text { for } p=q, \\
O\left(\frac{\ell}{d}\right) & \text { for } p \neq q .
\end{array}\right.
$$

The last estimate follows from the relation

$$
\left(\widehat{e}_{p}, \widehat{e}_{q}\right)_{L^{2}(\widehat{S})}=(4 \pi \hat{r})^{-1} \int_{\widehat{S}} e^{i k \beta \cdot\left(s_{p}-s_{q}\right)} d \beta=(4 \pi \hat{r})^{-1} \widehat{\jmath}\left(k\left(s_{p}-s_{q}\right)\right)
$$

and from the decay property (5.7) of $\widehat{\jmath}$ for $p \neq q$.

Remark 6. Contrary to the case of a closed mirror (compare Propositions 4.3 and 5.3 ), we have not been able to compare the spectral radius of $\hat{T}^{(1)}$ with the greatest value taken by the quantities $\left(\hat{r} C_{p}\right)^{2}$.

As in the case of a closed mirror, Proposition 5.3 shows that the eigenvectors of the time-reversal operator for an open mirror will produce selective focusing acoustic waves if

(i) the obstacles are small enough, compared to the wavelength,

(ii) the smallest distance between them is large, compared to the wavelength,

(iii) their capacities are all distinct.

Indeed in this case all the nonzero eigenvalues of $\widehat{T}^{(1)}$ will be simple: the diagonalization of the time-reversal operator will then yield approximations of the focusing densities $\widehat{e}_{p}$ since each $\widehat{e}_{p}$ generates an incident Herglotz wave $\widehat{u}_{I, p}$ which focuses on the $p$ th obstacle for

$$
\widehat{u}_{I, p}(x)=\int_{\widehat{S}} \widehat{e}_{p}(\alpha) u_{I}^{\alpha}(x) d \alpha=\frac{1}{\sqrt{4 \pi \widehat{r}}} \widehat{\jmath}\left(k\left(x-s_{p}\right)\right)=O\left(\frac{\ell}{\left\|x-s_{p}\right\|}\right) .
$$

Appendix A. Asymptotics for small obstacles. We detail here a constructive proof of the asymptotic behavior (4.2) of the scattering amplitude for small obstacles, claimed in Proposition 4.2. This result is formally derived in other papers (see, e.g., 
$[17,18])$. A more abstract proof based on potential theory was recently proposed in $[8]$.

The idea of our proof is to rewrite the scattering problem as a regular perturbation of a Fredholm equation in a fixed Hilbert space, in the sense that it does not depend on the size, say $\varepsilon$, of the obstacles:

$$
\left(I+\mathbb{K}^{\varepsilon}\right) \varphi^{\varepsilon}=g^{\varepsilon} .
$$

We obtain such a formulation by means of a variant of the integral method introduced by Jami and Lenoir [10], which has the advantage to involve nonsingular kernels, contrary to usual integral equations (for which perturbation theory requires more complicated arguments).

Consider the family of obstacles $\left\{\mathcal{O}_{p}^{\varepsilon} ; p=1, N\right\}$ introduced in subsection 4.2. We denote by $\Gamma_{p}^{\varepsilon}\left(\right.$ respectively, $\left.\Gamma_{p}\right)$ the boundary of $\mathcal{O}_{p}^{\varepsilon}$ (respectively, of $\left.\mathcal{O}_{p}\right), \Gamma^{\varepsilon}=$ $\bigcup_{p=1, N} \Gamma_{p}^{\varepsilon}$ and $\mathcal{O}^{\varepsilon}=\bigcup_{p=1, N} \mathcal{O}_{p}^{\varepsilon}$. Our exterior Dirichlet problem for the diffracted field $u^{\varepsilon}$ reads

$$
\left\{\begin{array}{l}
\Delta u^{\varepsilon}+k^{2} u^{\varepsilon}=0 \text { in } \mathbb{R}^{3} \backslash \overline{\mathcal{O}^{\varepsilon}}, \\
u^{\varepsilon}=f \text { on } \Gamma^{\varepsilon} \\
\text { R.C. }
\end{array}\right.
$$

where R.C. stands for the outgoing radiation condition, and $f=-u_{I}^{\alpha}$ is the Dirichlet datum associated with an incident plane wave $u_{I}^{\alpha}(x)=\exp (i k \alpha \cdot x)$ of direction $\alpha \in S^{2}$.

Reduction to a bounded domain. Around each reference obstacle $\mathcal{O}_{p}$, we delimit a bounded part $D_{p}$ of its exterior by a fictitious boundary $\Sigma_{p}$ which does not intersect $\Gamma_{p}$. We denote by $D_{p}^{\varepsilon}$ and $\Sigma_{p}^{\varepsilon}$ the images of $D_{p}$ and $\Sigma_{p}$ by the same dilation as for $\mathcal{O}_{p}^{\varepsilon}$, as well as $D^{\varepsilon}=\bigcup_{p=1, N} D_{p}^{\varepsilon}$ and $\Sigma^{\varepsilon}=\bigcup_{p=1, N} \Sigma_{p}^{\varepsilon}$.

The Jami-Lenoir method consists of introducing a transparent boundary condition on $\Sigma^{\varepsilon}$ which is derived from the usual integral representation of $u^{\varepsilon}$. Here, in order to get rid of the normal derivative of $u^{\varepsilon}$ on $\Gamma^{\varepsilon}$, the single-layer potential is re-expressed as a volume potential by Green's formula. Indeed it is easy to see that near $\Sigma^{\varepsilon}$ we have

$$
u^{\varepsilon}=f \stackrel{\Gamma^{\varepsilon}}{*} \frac{\partial G_{k}}{\partial \nu}+k^{2} u^{\varepsilon} \stackrel{D^{\varepsilon}}{*}\left(\chi^{\varepsilon} G_{k}\right)-\nabla u^{\varepsilon} \stackrel{D^{\varepsilon}}{*} \nabla\left(\chi^{\varepsilon} G_{k}\right),
$$

where the different "convolutions" represent, respectively, the surface double-layer potential

$$
\left\{f^{\Gamma^{\varepsilon}} \frac{\partial G_{k}}{\partial \nu}\right\}(x)=\int_{\Gamma^{\varepsilon}} f(y) \frac{\partial G_{k}}{\partial \nu_{y}}(x-y) d \gamma_{y}
$$

and the volume potentials

$$
\begin{aligned}
\left\{u^{\varepsilon}{ }^{D^{\varepsilon}}\left(\chi^{\varepsilon} G_{k}\right)\right\}(x) & =\int_{D^{\varepsilon}} u^{\varepsilon}(y) \chi^{\varepsilon}(y) G_{k}(x-y) d y \\
\left\{\nabla u^{\varepsilon}{ }^{D^{\varepsilon}} \nabla\left(\chi^{\varepsilon} G_{k}\right)\right\}(x) & =\int_{D^{\varepsilon}} \nabla u^{\varepsilon}(y) \cdot \nabla_{y}\left(\chi^{\varepsilon}(y) G_{k}(x-y)\right) d y .
\end{aligned}
$$

In the above expressions, $G_{k}$ stands for the outgoing Green function of $\Delta+k^{2}$, i.e., $G_{k}(x)=-\exp (i k|x|) /(4 \pi|x|)$, and $\chi^{\varepsilon}$ denotes a family of regular cutoff functions $\left(\chi_{p}^{\varepsilon}\right)_{p=1, \ldots, N}$ defined by the $\varepsilon$-dilation: $\chi_{p}^{\varepsilon}(x)=\chi_{p}\left(\left(x-s_{p}\right) / \varepsilon\right)$ if $x \in D_{p}^{\varepsilon}$, where each 
$\chi_{p}$ is equal to 1 in a vicinity of $\Gamma_{p}$ and 0 in a vicinity of $\Sigma_{p}$. Note that these integrals involve regular kernels when $x$ is near $\Sigma^{\varepsilon}$.

As a consequence if $u^{\varepsilon}$ solves (A.2), its restriction $v^{\varepsilon}$ to $D^{\varepsilon}$ satisfies

$$
\left\{\begin{array}{l}
\Delta v^{\varepsilon}+k^{2} v^{\varepsilon}=0 \text { in } D^{\varepsilon} \\
v^{\varepsilon}=f \text { on } \Gamma^{\varepsilon} \\
Z^{\varepsilon} v^{\varepsilon}=Z^{\varepsilon}\left\{f^{\Gamma^{\varepsilon}} \frac{\partial G_{k}}{\partial \nu}+k^{2} v^{\varepsilon}{ }^{D^{\varepsilon}}\left(\chi^{\varepsilon} G_{k}\right)-\nabla v^{\varepsilon}{ }^{D^{\varepsilon}} \nabla\left(\chi^{\varepsilon} G_{k}\right)\right\} \text { on } \Sigma^{\varepsilon},
\end{array}\right.
$$

where $Z^{\varepsilon}$ stands for the boundary operator $(\partial / \partial \nu+i / \varepsilon)$ on $\Sigma^{\varepsilon}$.

Conversely, the solution to this problem extends outside $\Sigma^{\varepsilon}$ (by the integral representation) to the solution to (A.2) (thanks to the term involving $i / \varepsilon$ which prevents the so-called irregular frequencies from being real; see [10]).

The limiting process. In order to work in a functional framework independent of $\varepsilon$, we perform in each subdomain $D_{p}^{\varepsilon}$ the change of variable $\xi=\left(x-s_{p}\right) / \varepsilon$. By denoting $\varphi_{p}^{\varepsilon}(\xi)=v^{\varepsilon}(x)$ and $f_{p}^{\varepsilon}(\xi)=f(x)$, for $x \in D_{p}^{\varepsilon}$, as well as

$$
G_{p q}^{\varepsilon}(\xi, \eta)=G_{k}\left(s_{p}-s_{q}+\varepsilon(\xi-\eta)\right) \text { for } \xi \in \overline{D_{p}} \text { and } \eta \in \overline{D_{q}}
$$

problem (A.3) amounts to a family of $N$ problems set on the domains $D_{p}$ coupled by the transparent boundary conditions written on $\Sigma_{p}$ :

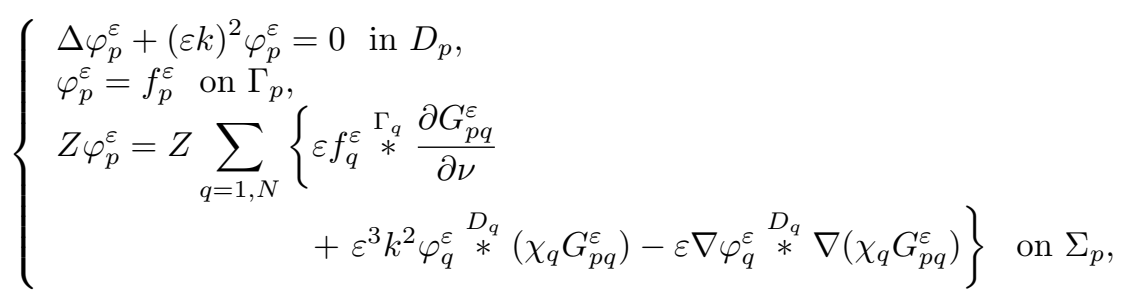

where $Z=(\partial / \partial \nu+i)$ on $\Sigma_{p}$.

We are now able to define the formal limit of the latter problem. Let $G_{0}$ be the limit of $G_{\varepsilon k}$ when $\varepsilon$ tends to 0, i.e., $G_{0}(x)=-1 /(4 \pi|x|)$. Notice that

$$
\begin{array}{ll}
G_{p q}^{\varepsilon}(\xi, \eta)=G_{k}\left(s_{p}-s_{q}\right)+O(\varepsilon) & \text { if } p \neq q, \\
G_{p p}^{\varepsilon}(\xi, \eta)=\varepsilon^{-1} G_{0}(\xi-\eta)+O(1) & \text { if } p=q,
\end{array}
$$

where these formulas hold uniformly in any compact subset of $\overline{D_{p}} \times \overline{D_{q}}$ which does not contain points of the diagonal when $p=q$, and can be derived with respect to $\xi$ or $\eta$. Hence the formal limit of problem (A.4) reads as

$$
\left\{\begin{array}{l}
\Delta \varphi_{p}^{0}=0 \text { in } D_{p}, \\
\varphi_{p}^{0}=f_{p}^{0}=-e^{i k \alpha \cdot s_{p}} \text { on } \Gamma_{p}, \\
Z \varphi_{p}^{0}=Z\left\{f_{p}^{0}{ }^{\Gamma_{p}} \stackrel{\partial G_{0}}{\partial \nu}-\nabla \varphi_{p}^{0} \stackrel{D_{p}}{*} \nabla\left(\chi_{p} G_{0}\right)\right\} \text { on } \Sigma_{p}
\end{array}\right.
$$

which correspond to a family of uncoupled problems. Each of them amounts to solving an exterior Laplace equation. More precisely, we can write that $\varphi_{p}^{0}=-u_{I}^{\alpha}\left(s_{p}\right) V_{p}$, where $V_{p}$ is the static potential solution to

$$
\left\{\begin{array}{l}
\Delta V_{p}=0 \text { in } \mathbb{R}^{3} \backslash \mathcal{O}_{p} \\
V_{p}=1 \text { on } \Gamma_{p} \\
V_{p}=O(1 / x) \text { as }|x| \rightarrow \infty
\end{array}\right.
$$


Convergence. Consider the closed subspace of the usual Sobolev space $H^{1}\left(D_{p}\right)$ given by $\mathcal{H}_{p}=\left\{\psi_{p} \in H^{1}\left(D_{p}\right) ; \psi_{p}=0\right.$ on $\left.\Gamma_{p}\right\}$. The variational formulation of (A.4) appears as a coupled system of variational equations:

$$
\begin{aligned}
& \text { Find } \varphi_{p}^{\varepsilon} \in f_{p}^{\varepsilon}+\mathcal{H}_{p}, p=1, N, \text { such that } \\
& \int_{D_{p}} \nabla \varphi_{p}^{\varepsilon} \cdot \overline{\nabla \psi_{p}}-(\varepsilon k)^{2} \int_{D_{p}} \varphi_{p}^{\varepsilon} \overline{\psi_{p}}+i \int_{\Sigma_{p}} \varphi_{p}^{\varepsilon} \overline{\psi_{p}} d \sigma \\
& +\int_{\Sigma_{p}} Z\left\{\sum_{q=1, N} \varepsilon^{3} k^{2} \varphi_{q}^{\varepsilon}{ }^{D_{q}}\left(\chi_{q} G_{p q}^{\varepsilon}\right)-\varepsilon \nabla \varphi_{q}^{\varepsilon}{ }^{D_{q}} \nabla\left(\chi_{q} G_{p q}^{\varepsilon}\right)\right\} \overline{\psi_{p}} d \sigma \\
& =\int_{\Sigma_{p}} Z\left\{\sum_{q=1, N} \varepsilon f_{q}^{\varepsilon} *^{\Gamma_{q}} \frac{\partial G_{p q}^{\varepsilon}}{\partial \nu}\right\} \overline{\psi_{p}} d \sigma \quad \forall \psi_{p} \in \mathcal{H}_{p}, p=1, N .
\end{aligned}
$$

Adding these equations yields the announced Fredholm equation (A.1) in the Hilbert space $\mathcal{H}=\mathcal{H}_{1} \times \cdots \times \mathcal{H}_{N}$ which can be equipped with the scalar product

$$
(\varphi, \psi)=\sum_{p=1, N} \int_{D_{p}} \nabla \varphi_{p} \cdot \overline{\nabla \psi_{p}}
$$

Indeed, define $\varphi^{\varepsilon}=\left(\varphi_{1}^{\varepsilon}, \ldots, \varphi_{N}^{\varepsilon}\right), f^{\varepsilon}=\left(f_{1}^{\varepsilon}, \ldots, f_{N}^{\varepsilon}\right)$ and, respectively, the operator $\mathbb{K}^{\varepsilon}$ defined in $\mathcal{H}$ and $g^{\varepsilon} \in \mathcal{H}$ by

$$
\begin{aligned}
\left(\mathbb{K}^{\varepsilon} \varphi, \psi\right) & =\sum_{p=1, N}-(\varepsilon k)^{2} \int_{D_{p}} \varphi_{p} \overline{\psi_{p}}+i \int_{\Sigma_{p}} \varphi_{p} \overline{\psi_{p}} d \sigma \\
& +\int_{\Sigma_{p}} Z\left\{\sum_{q=1, N} \varepsilon^{3} k^{2} \varphi_{q}{ }^{D_{q}}\left(\chi_{q} G_{p q}^{\varepsilon}\right)-\varepsilon \nabla \varphi_{q} *^{D_{q}} \nabla\left(\chi_{q} G_{p q}^{\varepsilon}\right)\right\} \overline{\psi_{p}} d \sigma \\
\left(g^{\varepsilon}, \psi\right) & =\sum_{p=1, N} \int_{\Sigma_{p}} Z\left\{\sum_{q=1, N} \varepsilon f_{q}^{\varepsilon} *^{\Gamma_{q}} \frac{\partial G_{p q}^{\varepsilon}}{\partial \nu}\right\} \overline{\psi_{p}} d \sigma
\end{aligned}
$$

for all $\varphi=\left(\varphi_{1}, \ldots, \varphi_{N}\right)$ and $\psi=\left(\psi_{1}, \ldots, \psi_{N}\right)$ in $\mathcal{H}$. Then our coupled system reads as follows:

$$
\text { Find } \varphi^{\varepsilon} \in f^{\varepsilon}+\mathcal{H} \text { such that }\left(I+\mathbb{K}^{\varepsilon}\right) \varphi^{\varepsilon}=g^{\varepsilon} .
$$

And of course we have a similar expression of the limit problem (A.6) with

$$
\begin{aligned}
\left(\mathbb{K}^{0} \varphi, \psi\right) & =\sum_{p=1, N} i \int_{\Sigma_{p}} \varphi_{p} \overline{\psi_{p}} d \sigma-\int_{\Sigma_{p}} Z\left\{\nabla \varphi_{p}{ }^{D_{p}} \nabla\left(\chi_{q} G_{0}\right)\right\} \overline{\psi_{p}} d \sigma \\
\left(g^{0}, \psi\right) & =\sum_{p=1, N} \int_{\Sigma_{p}} Z\left\{f_{p}^{0} \stackrel{\Gamma_{p}}{*} \frac{\partial G_{0}}{\partial \nu}\right\} \overline{\psi_{p}} d \sigma .
\end{aligned}
$$

Note that the uniqueness of the solution to (A.2) (respectively, (A.7)) implies that $I+\mathbb{K}^{\varepsilon}$ (respectively, $I+\mathbb{K}^{0}$ ) is injective, and thus bijective thanks to the following.

Lemma A.1. $\mathbb{K}^{\varepsilon}$ defines a family of compact operators in $\mathcal{H}$ which satisfies

$$
\left\|\mathbb{K}^{\varepsilon}-\mathbb{K}^{0}\right\|=\sup _{\varphi, \psi \in \mathcal{H} \backslash\{0\}} \frac{\left.\left(\mathbb{K}^{\varepsilon}-\mathbb{K}^{0}\right) \varphi, \psi\right)}{\|\varphi\|\|\psi\|}=O(\varepsilon) .
$$


Proof. Consider, for instance, the part of $\mathbb{K}^{\varepsilon}$ corresponding to the operator $\mathbb{T}_{p q}^{\varepsilon}$ given by

$$
\begin{aligned}
\left(\mathbb{T}_{p q}^{\varepsilon} \varphi, \psi\right) & =\int_{\Sigma_{p}} Z\left\{\varepsilon \nabla \varphi_{q}{ }^{D_{q}} \nabla\left(\chi_{q} G_{p q}^{\varepsilon}\right)\right\} \overline{\psi_{p}} d \sigma \\
& =\int_{\Sigma_{p}} \int_{D_{q}} \nabla \varphi_{q}(\eta) \cdot \nabla_{\eta}\left(\varepsilon \chi_{q}(\eta) Z_{\xi} G_{p q}^{\varepsilon}(\xi-\eta)\right) d \eta \overline{\psi_{p}} d \sigma_{\xi} .
\end{aligned}
$$

We detail the proof only for the latter; similar arguments can be used for the other terms involved in the definition of $\mathbb{K}^{\varepsilon}$.

The compactness of $\mathbb{T}_{p q}^{\varepsilon}$ can be easily deduced from that of its adjoint. Indeed, using Schwarz inequality yields

$$
\begin{aligned}
\left\|\left(\mathbb{T}_{p q}^{\varepsilon}\right)^{*} \psi\right\| & =\sup _{\varphi \in \mathcal{H} \backslash\{0\}} \frac{\left(\mathbb{T}_{p q}^{\varepsilon} \varphi, \psi\right)}{\|\varphi\|} \leq C_{p q}^{\varepsilon}\|\psi\|_{L^{2}\left(\Sigma_{p}\right)}, \text { where } \\
C_{p q}^{\varepsilon} & =\left(\int_{\Sigma_{p}} \int_{D_{q}}\left\|\nabla_{\eta}\left\{\varepsilon \chi_{q}(\eta) Z_{\xi} G_{p q}^{\varepsilon}(\xi-\eta)\right\}\right\|^{2} d \eta d \sigma_{\xi}\right)^{1 / 2} .
\end{aligned}
$$

But the trace operator is compact from $H^{1}\left(D_{q}\right)$ to $L^{2}\left(\Sigma_{p}\right)$, which implies the compactness of $\left(\mathbb{T}_{p q}^{\varepsilon}\right)^{*}$ in $\mathcal{H}$.

If $p \neq q$, formula (A.5) shows that $C_{p q}^{\varepsilon}=O(\varepsilon)$, and consequently the same holds for $\left\|\mathbb{T}_{p q}^{\varepsilon}\right\|=\left\|\left(\mathbb{T}_{p q}^{\varepsilon}\right)^{*}\right\|$. If $p=q$, the limit operator is given by

$$
\left(\mathbb{T}_{p p}^{0} \varphi, \psi\right)=\int_{\Sigma_{p}} Z\left\{\nabla \varphi_{p}{ }^{D_{p}} \nabla\left(\chi_{p} G_{0}\right)\right\} \overline{\psi_{p}} d \sigma
$$

since (A.5) shows in this case (again by Schwarz inequality) that

$$
\left|\left(\left(\mathbb{T}_{p p}^{\varepsilon}-\mathbb{T}_{p p}^{0}\right) \varphi, \psi\right)\right| \leq \varepsilon C\|\nabla \varphi\|_{L^{2}\left(D_{p}\right)}\|\psi\|_{L^{2}\left(\Sigma_{p}\right)} .
$$

Hence $\left\|\mathbb{T}_{p p}^{\varepsilon}-\mathbb{T}_{p p}^{0}\right\|=O(\varepsilon)$.

Lemma A.1 turns our problem into one of the simplest situations of perturbation theory [11]: the use of the Neumann series readily shows that

$$
\left\|\left(I+\mathbb{K}^{\varepsilon}\right)^{-1}-\left(I+\mathbb{K}^{0}\right)^{-1}\right\|=O(\varepsilon) .
$$

It remains to notice that $\left\|f^{\varepsilon}-f^{0}\right\|$ and $\left\|g^{\varepsilon}-g^{0}\right\|$ are also of order $\varepsilon$, from which we conclude that

$$
\left\|\varphi^{\varepsilon}-\varphi^{0}\right\|=O(\varepsilon)
$$

The scattering amplitude. Thanks to formula (2.2), the local convergence expressed by the latter result also provides the far field asymptotics. Here, using our homothetic changes of variables, the scattering amplitude reads

$$
A^{\varepsilon}(\alpha, \beta)=\frac{-\varepsilon}{4 \pi} \sum_{p=1, N} \int_{\Gamma_{p}} \frac{\partial}{\partial \nu}\left(\varphi_{p}^{\varepsilon}(\alpha)-f_{p}^{\varepsilon}(\alpha)\right) \overline{f_{p}^{\varepsilon}(\beta)} d \gamma
$$

On one hand, (A.10) implies that $\partial \varphi_{p}^{\varepsilon}(\alpha) / \partial \nu$ tends to $\partial \varphi_{p}^{0}(\alpha) / \partial \nu=-u_{I}^{\alpha}\left(s_{p}\right) \partial V_{p} / \partial \nu$ in $H^{-1 / 2}\left(\Gamma_{p}\right)$ (recall that $V_{p}$ is defined in (A.7)). On the other hand, $f_{p}^{\varepsilon}(\alpha)$ tends to 
the constant function $f_{p}^{0}(\alpha)=-u_{I}^{\alpha}\left(s_{p}\right)$. Hence

$$
\begin{aligned}
A^{\varepsilon}(\alpha, \beta) & =\frac{-\varepsilon}{4 \pi} \sum_{p=1, N} C_{p} u_{I}^{\alpha}\left(s_{p}\right) \overline{u_{I}^{\beta}\left(s_{p}\right)}+O\left(\varepsilon^{2}\right), \text { where } \\
C_{p} & =\int_{\Gamma_{p}} \frac{\partial V_{p}}{\partial \nu} d \gamma=\int_{\mathbb{R}^{3} \backslash \mathcal{O}_{p}}\left|\nabla V_{p}\right|^{2}
\end{aligned}
$$

is referred to as the capacity of the obstacle $\mathcal{O}_{p}$. Proposition 4.2 is thus proved.

Acknowledgments. The authors would like to thank the referees for their valuable comments and suggestions.

\section{REFERENCES}

[1] M. Abramowitz and I. A. Stegun, Pocketbook of Mathematical Functions, Abridged edition of Handbook of Mathematical Functions, Verlag Harri Deutsch, Thun, 1984.

[2] C. Bardos And M. Fink, Mathematical foundations of the time reversal mirror, Asymptot. Anal., 29 (2002), pp. 157-182.

[3] M. Cheney, The linear sampling method and the MUSIC algorithm, Inverse Problems, 17 (2001), pp. 591-595.

[4] M. Cheney, D. IsaAcson, and M. Lassas, Optimal acoustic measurements, SIAM J. Appl. Math., 61 (2001), pp. 1628-1647.

[5] D. Colton and R. Kress, Eigenvalues of the far field operator and inverse scattering theory, SIAM J. Math. Anal., 26 (1995), pp. 601-615.

[6] D. Colton and R. KRess, Eigenvalues of the far field operator for the Helmholtz equation in an absorbing medium, SIAM J. Appl. Math., 55 (1995), pp. 1724-1735.

[7] D. Colton and R. Kress, Inverse Acoustic and Electromagnetic Scattering Theory, SpringerVerlag, Berlin, 1992.

[8] Y. Dermenjian and E. Jalade, Behaviour of $\left(-\Delta-k^{2}-i 0^{+}\right)^{-1}$ outside fading obstacles, independent scattering hypothesis and applications, Math. Methods Appl. Sci., 26 (2003), pp. 1075-1092.

[9] M. Fink, Time-reversal in acoustics, Contemporary Physics, 37 (1996), pp. 95-109.

[10] A. JAmi AND M. LenOIR, A variational formulation for exterior problems in linear hydrodynamics, Comput. Methods Appl. Mech. Engrg., 16 (1978), pp. 341-359.

[11] T. Kato, Perturbation Theory for Linear Operators, Springer-Verlag, New York, 1966.

[12] A. KIRSCH, The MUSIC-Algorithm and the factorization method in inverse scattering theory for inhomogeneous media, Inverse Problems, 18 (2002), pp. 1025-1040.

[13] P. D. Lax And R. S. Phillips, Scattering Theory, Academic Press, New York, 1967.

[14] T. D. Mast, A. I. Nachman, And R. C. WAAG, Focusing and imaging using the eigenfunctions of the scattering operator, J. Acoust. Soc. Am., 102 (1997), pp. 715-725.

[15] P. Blomgren, G. Papanicolaou, and H. Zhao, Super-resolution in time-reversal acoustics, J. Acoust. Soc. Am., 111 (2002), pp. 230-248.

[16] C. Prada And M. Fink, Eigenmodes of the time-reversal operator: A solution to selective focusing in multiple-target media, Wave Motion, 20 (1994), pp. 151-163.

[17] A. G. Ramm, Wave scattering by small bodies, Rep. Math. Phys., 21 (1985), pp. 69-77.

[18] A. G. Ramm, Scattering by Obstacles, Mathematics and its Applications, D. Reidel, Dordrecht, 1986.

[19] E. M. Stein, Oscillatory integrals in Fourier analysis, in Beijing Lectures in Harmonic Analysis, Ann. of Math. Stud. 112, E. M. Stein, ed., Princeton University Press, Princeton, NJ, 1986, pp. 307-355.

[20] A. C. ZaAnen, Linear Analysis. Measure and Integral, Banach and Hilbert Space Linear Integral Equations, North-Holland, Amsterdam, 1953. 